\title{
A eficácia da terapia quiroprática no tratamento da escoliose idiopática do adolescente (EIA): uma revisão integrativa
}

\section{The effectiveness of chiropractic therapy in the treatment of adolescent idiopathic scoliosis (AIS): an integrative review}

\author{
Lídya Maria Calisto Viana ${ }^{1}$, Keven do Nascimento Pereira ${ }^{1}$, Tassiane Maria Alves Pereira ${ }^{2}$, Janaína de Moraes \\ Silva ${ }^{* 1,3}$
}

Como citar esse artigo. Viana

L.M.C; Pereira, K.N; Pereira, T.M.A;

Silva, J.M. A eficácia da terapia quiroprática no tratamento da escoliose idiopática do adolescente (EIA): uma revisão integrativa. Reista de Saúde. 2021 Abr./Jul.; 12 (2): 17-21.

\section{Resumo}

A Escoliose Idiopática do Adolescente (EIA) é atualmente caracterizada por curvatura da coluna acima de 10 graus, seguida de rotação vertebral coexistente, sendo uma doença que atinge crianças e adolescentes, correspondendo a cerca de $5,2 \%$ da população. Incorporada como uma técnica alternativa, a quiropraxia busca avaliar e tratar as desordens que afetam o alinhamento dos músculos e ossos do corpo, possuindo grande relação com a redução da curvatura da coluna vertebral, exercendo atribuições importantes no monitoramento e ajuste da escoliose na fase inicial. O objetivo desta revisão foi verificar na literatura, evidências sobre a aplicação da quiropraxia no tratamento da EIA e avaliar seus efeitos na redução da progressão das curvas. Assim, com base nos critérios rigorosamente estabelecidos, foi realizado uma busca nas bases de dados PubMed, Semantic Scholar, Web of Science, Scopus, PEDRo e Science Direct, envolvendo estudos de casos, coorte e ensaios clínicos publicados, originalmente na língua inglesa, dos últimos 10 anos, durante os meses de fevereiro e março de 2021. Foram incluídos 6 artigos científicos, nas quais evidenciaram a redução do ângulo de Cobb como o principal elemento observado diante do tratamento da EIA, proporcionando estabilidade da coluna, melhora da dor e diminuição da curvatura espinhal. Com base nos resultados, foi demonstrado que a quiropraxia é uma estratégia importante nos primeiros meses de tratamento, embora não sendo possível concluir sobre a eficácia desta intervenção, devido a quantidade reduzida e a heterogeneidade dos estudos, espera-se que outras análises possam ser realizadas, permitindo preencher ainda mais esta lacuna científica.

Palavras-chave: Quiropraxia; Escoliose idiopática; Adolescentes.

\begin{abstract}
Adolescent Idiopathic Scoliosis (AIS) is currently characterized by curvature of the spine above 10 degrees, followed by coexistent vertebral rotation, and is a disease that affects children and adolescents, corresponding to about $5.2 \%$ of the population. Incorporated as an alternative technique, chiropractic care seeks to evaluate and treat disorders that affect the alignment of the muscles and bones of the body, and is closely related to the reduction of spinal curvature, exercising important functions in the monitoring and adjustment of scoliosis in its initial phase. The objective of this review was to verify in the literature, evidence on the application of chiropractic care in the treatment of AIS, and to evaluate its effects in reducing the progression of the curves. Thus, based on the rigorously established criteria, a search was conducted in PubMed, Semantic Scholar, Web of Science, Scopus, PEDRo and Science Direct databases, involving case studies, cohort and clinical trials published, originally in the English language, from the last 10 years, during the months of February and March 2021. Six scientific articles were included, which showed the reduction of the Cobb angle as the main element observed in the treatment of AIS, providing stability of the spine, improvement of pain and reduction of spinal curvature. Based on the results, it was shown that chiropractic care is an important strategy in the first months of treatment, although it is not possible to conclude on the effectiveness of this intervention, due to the small number and heterogeneity of the studies, it is hoped that other analyses can be performed, allowing this scientific gap to be filled even more.
\end{abstract}

Keywords: Chiropractic; Idiopathic scoliosis; Adolescents.

\section{Introdução}

A escoliose é o distúrbio espinhal mais recorrente entre os adolescentes ${ }^{1}$. Dentre seus tipos, destacamse a congênita, neuromuscular ou idiopática; desta classificação, aproximadamente $85 \%$ dos casos são idiopáticos ${ }^{2}$. Esse tipo é subcategorizado da seguinte forma: escoliose infantil (0-3 anos de idade), escoliose juvenil (4-10 anos de idade), escoliose adolescente (1118 anos de idade),e escoliose adulta (idade $>18$ anos) ${ }^{3}$.
A Escoliose Idiopática do Adolescente (EIA) é uma doença que afeta a coluna vertebral e atinge até 5,2\% da população. É caracterizada pela curvatura da coluna vertebral acima de dez graus, acompanhada de rotação vertebral concomitante ${ }^{4}$. Apesar de se apresentar como benigna na maior parte dos casos, o seu desenvolvimento pode acarretar alterações significativas da morfologia corporal, diminuição do volume torácico, respiração prejudicada, elevada dor nas costas, além de graves problemas estéticos ${ }^{5}$. Assim, mesmo quando não há uma

\footnotetext{
Afiliação dos autores:

${ }^{1}$ Universidade Estadual do Piauí - UESPI, Teresina-PI, Brasil; lidyacalistoviana@gmail.com. https://orcid.org/0000-0001-7621-138X.

${ }^{2}$ Universidade Estadual do Piauí - UESPI, Teresina-PI, Brasil; kevennascimento08@gmail.comhttps://orcid.org/0000-0003-2392-9130.

${ }^{3}$ Centro Universitário UNINASSAU, Teresina - PI, Brasil;Pós-graduada em Fisioterapia Hospitalar pela Inspirar, Teresina-PI, Brasil; Mestranda em Biotecnologia - UFPI/UFDPAR, Parnaíba-PI, Brasil; tassiane.alves07@gmail.comhttps://orcid.org/0000-0002-8876-7438.

${ }^{4}$ Doutora em Engenharia Biomédica pela UNIVAP, Pós-doutorado em Ciências Biomédicas - UFPI, Parnaíba-PI, Brasil; fisiojanainams@gmail.com; https://orcid.org/0000-00028693-3957

* Email de correspondência: fisiojanainams@gmail.com

Recebido em: 17/11/20. Aceito em: 07/05/21.
} 
causa clara, é comum a ocorrência de deformidades da coluna vertebral na adolescência e, uma vez iniciada a deformidade, a curvatura da coluna se torna mais grave progressivamente, dessa forma, podendo evoluir para a escoliose, cuja taxa de prevalência é de $0,3 \%$ a $21 \%$.

O risco de progressão dessa curva espinhal pode ser induzido por inúmeros fatores como a capacidade de crescimento dos indivíduos em termos de idade da menarca e maturidade esquelética, a magnitude da curva e o tipo da mesma ${ }^{7}$. Conforme a maturidade esquelética aumenta, surge a redução do risco de progressão. Entretanto, curvas maiores ainda tendem a se agravar após alcançar a maturidade esquelética ${ }^{8}$.

Esse distúrbio espinhal ocorre lenta e gradualmente, portanto, é difícil obter um diagnóstico até que ela se desenvolva totalmente ${ }^{6}$. Diante dos vários problemas de saúde que aparecem quando a escoliose avança, a dor nas costas prevalece entre os indivíduos acometidos com essa enfermidade quando se comparada com os que não a possui ${ }^{9}$. A conscientização sobre a necessidade de diagnóstico e tratamento conservador precoce da escoliose aumenta, uma vez que já em 1981, foi descoberto na Suécia que os exames e tratamentos podem retardar o progresso da escoliose de $40 \%$ para $63 \%{ }^{6}$.

Em relação ao tratamento, é importante apontar que ele se faz necessário tanto para promoção da saúde, como para prevenir reações adversas futuras ${ }^{10}$. Existem diversas metodologias para tratar a EIA com o objetivo de reduzir a progressão, estabilização e diminuição da magnitude da curva por fisioterapia, exercícios, cirurgia, tração, órtese, moldagem, biofeedback e observação ${ }^{11}$. As formas de tratamento convencionais para escoliose idiopática do adolescente envolvem a observação de curvas abaixo de $20^{\circ}$, órteses para curvas entre $20^{\circ}$ a $40^{\circ}$ e cirurgia acima de $50^{\circ}{ }^{12}$. Dessa forma, qualquer que seja o tratamento escolhido, o objetivo principal é sempre interromper a progressão da curva e evitar a cirurgia. Estudos demonstraram que técnicas conservadoras de intervenção não cirúrgica aumentam a qualidade de vida nesses pacientes, aumentando a função pulmonar, força da coluna vertebral, mobilidade, diminuição da dor e melhora da imagem corporal ${ }^{11}$.

Nesse sentido, a quiropraxia é incorporada como uma técnica alternativa que avalia e trata as desordens que afetam o alinhamento dos músculos e ossos do corpo $^{13}$. Em 1895, nos Estados Unidos, a medicina quiroprática ficou conhecida como a profissão da cura.Originalmente criada sob o ideal de que os desalinhamentos da coluna vertebral, chamados subluxações vertebrais, poderiam atingir de forma negativa os nervos periféricos adjacentes e resultar em futuros distúrbios naturais de saúde, se tornou um forte aliado na diminuição da curvatura espinhal, desempenhando papel no monitoramento e ajuste da escoliose no estágio inicial, contribuindo para o alívio da dor, desconforto e melhoria da estética, através da mobilização de regiões da coluna inacessíveis por outros métodos ${ }^{14}$.

Diante disso, o presente estudo teve como objetivo, verificar na literatura, a eficácia da aplicação da quiropraxia no tratamento da Escoliose Idiopática em Adolescentes (EIA) e avaliar seus efeitos na redução da progressão das curvas.

\section{Material e Métodos}

Trata-se de um estudo de revisão integrativa da literatura, realizadanas bases de dados PubMed, Semantic Scholar, Web of Science, Scopus, PEDRoe Science Direct. entre os meses de fevereiro e março de 2021. Na busca dos artigos científicos, foi utilizado os seguintes descritores, segundo os "Descritores em Ciência da Saúde" -DeCs - Chiropractic;Scoliosis;Adolescent. Ademais, aplicou-se a combinação das subsequentes palavras chaves: "chiropractic" "idiopathicscoliosis" e "adolescent", associadas aos termos "chiropractic AND idiopathicscoliosis AND adolescent".

Para tanto, foram percorridas três etapas: 1) Identificação do tema, definição da questão da esquisa e criação do protocolo de pesquisa; 2) Aplicação dos critérios de elegibilidade, assim como a busca na literatura; 3) Extração e sintetização das informações dos estudos de acordo com o objetivo da pesquisa;

Foram incluídos em análise, artigos publicados originalmente na língua inglesa envolvendo estudos de casos, coorte e ensaios clínicos publicados nos últimos 10 anos, e excluídos aqueles que o tipo de estudo não é compatível; artigos duplicados nas bases de dados selecionadas; e artigos que compararam a quiropraxia com outro meio de intervenção.

Inicialmente, dois revisores independentes identificaram os estudos publicados e que seguiam de acordo com os títulos e resumos. Em seguida, os resumos analisados foram selecionados de acordo com os critérios estipulados.

\section{Resultados}

Nessa pesquisa bibliográfica, foram incluídos seis artigos científicos elegíveis que seguiram rigorosamente os critérios previamente estabelecidos. Para análise da pesquisa, apresenta-se o fluxograma (Figura 1) com o objetivo de examinar a etapa de verificação, sendo encontrados 1318 artigos de acordo com as bases de dados escolhidas, seguindo os critérios de inclusão e exclusão e por fim, integrando os seis artigos para a composição desta revisão integrativa. A seguir, o fluxograma que remete a pesquisa dos artigos científicos realizada em detalhes: 


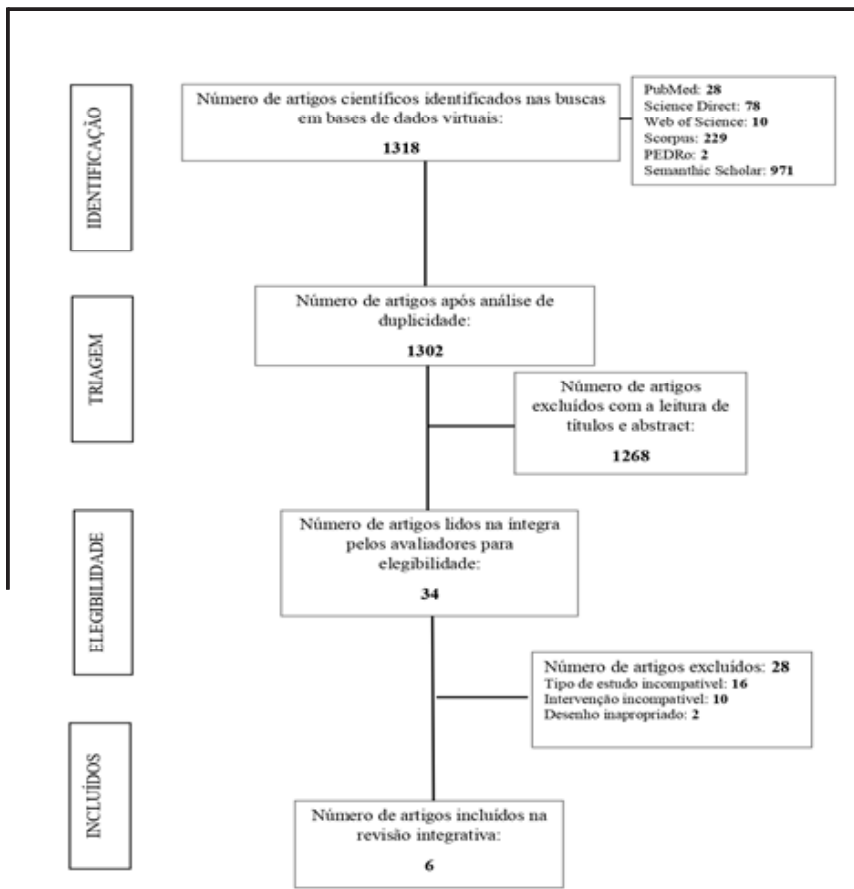

Figura 1. Fluxograma da seleção dos artigos a serem incluídos no estudo.

As informações contidas na Tabela 1 apresentam o sumário das características dos estudos incluídos, de acordo com o título, intervenção, resultados e conclusão.

\section{Discussão}

$\mathrm{Na}$ presente revisão integrativa, verificou-se as manobras quiropráticas, buscando a eficácia diante dos estudos incluídos. Desde então, apresentou-se a tabela 1, desenvolvida a partir da síntese dos artigos integrados, na qual descrevem a redução do ângulo de $\mathrm{Cobb}^{16}$, diminuição da estabilidade da coluna ${ }^{6 ; 17}$, melhora da dor e a correção da curvatura espinhal $1^{4 ; 15 ; 17}$. Com relação aos estudos que foram apresentados, a redução do ângulo de Cobb é o principal elemento observado diante do tratamento da Escoliose Idiopática do Adolescente (EIA), expondo resultados satisfatórios quanto à intervenção. Nesse sentido, é importante observar o valor do ângulo de Cobb antes de iniciar qualquer tratamento, sendo que em valores abaixo de $20^{\circ}$, é possível manter uma boa qualidade de vida, sem haver prejuízos cinéticos-funcionais ${ }^{12}$.

A predominância da EIA atinge $\mathrm{o}$ sexo feminino que abrange a faixa etária dos 10 aos 15 anos de idade. Ainda que ambos os sexos sejam afetados, as fêmeas possuem maiores chances de avançar para um estágio que requer tratamento. $\mathrm{O}$ diagnóstico precoce

Tabela 1. Apresentação das informações contidas nos artigos selecionados na Revisão Integrativa.

\begin{tabular}{|c|c|}
\hline TÍTULO & INTERVENÇÃO \\
\hline $\begin{array}{l}\text { Results of chiro- } \\
\text { practic scoliosis } \\
\text { rehabilitation treat- } \\
\text { ment at two years } \\
\text { post-skeletal matu- } \\
\text { rity in identical fe- } \\
\text { male twins }{ }^{16} \text {. }\end{array}$ & $\begin{array}{l}\text { Foram realizadas } 3 \text { visitas por dia durante } 2 \text { sema- } \\
\text { nas. As gêmeas foram submetidas à distração mo- } \\
\text { torizada da flexão, tração motorizada da extensão } \\
\text { cervical e cadeira de tração para escoliose. }\end{array}$ \\
\hline $\begin{array}{l}\text { The effect of chiro- } \\
\text { practic techniques } \\
\text { on the Cobb angle in } \\
\text { idiopathic scoliosis } \\
\text { arising in adoles- } \\
\text { cence }^{6} \text {. }\end{array}$ & $\begin{array}{l}\text { O ângulo de Cobb foi medido através de raio-X. } \\
\text { Uma massagem de tecidos moles foi realizada } \\
\text { antes da quiropraxia, a fim de evitar lesões. Uti- } \\
\text { lizaram técnicas de correção de processos ilíacos } \\
\text { e mamilares, vertebral torácica e cervicais. A cor- } \\
\text { reção foi administrada por } 30 \text { min por sessão, } 3 \\
\text { vezes por semana durante } 8 \text { semanas. }\end{array}$ \\
\hline $\begin{array}{l}\text { Chiropractic Reha- } \\
\text { bilitation for Ado- } \\
\text { lescent Idiopathic } \\
\text { Scoliosis: End-of- } \\
\text { Growth and Skeletal } \\
\text { Maturity Results }{ }^{17} \text {. }\end{array}$ & $\begin{array}{l}\text { Os pacientes realizaram equilíbrio proprioceptivo } \\
\text { no disco vestibular, tração repetitiva motorizada, } \\
\text { tração posicional supina e manipulação quiroprá- } \\
\text { tica de acordo com cada padrão de curva. Foram } \\
\text { feitas uma média de } 1-3 \text { manipulações ao longo } \\
\text { de } 1-2 \text { semanas de terapia. }\end{array}$ \\
\hline $\begin{array}{l}\text { Bridging the gap be- } \\
\text { tween observation } \\
\text { and brace treatment } \\
\text { for adolescent idio- } \\
\text { pathic scoliosis }{ }^{15} \text {. }\end{array}$ & $\begin{array}{l}\text { Realizaram Teste de Adams, os tratamentos qui- } \\
\text { ropráticos consistiram em massagem geral apli- } \\
\text { cada aos músculos posturais. Houve distração da } \\
\text { flexão com o paciente em decúbito frontal, após } \\
\text { uma tração intermitente na coluna vertebral. De- } \\
\text { pois do procedimento anterior, a manipulação da } \\
\text { coluna foi exercida por } 10 \text { minutos. }\end{array}$ \\
\hline
\end{tabular}

RESULTADOS

CONCLUSÃO

O ângulo de Cobb reduziu de $22^{\circ}$ para $19^{\circ}$ na paciente $\mathrm{A}$, e de $26^{\circ}$ para $16^{\circ}$ na paciente $\mathrm{B}$.

As técnicas de quiropraxia apresentaram um resultado significativo na redução do ângulo de Cobb dos pacientes em menos de 4 semanas de intervenção.

O tratamento trouxe resultados positivos em $90 \%$ dos pacientes. Dentre eles $51,7 \%$ tiveram correção da curva e 38,3\% apresentaram estabilização da curva.

O Teste de Adams apontou positivo; O tratamento durou 6 meses e durante o acompanhamento de 9 meses, os ângulos de Cobb foi medido através de radiografias frequentes que registraram a redução de $6^{\circ}$ na curva torácica (de $26^{\circ}$ para $20^{\circ}$ ) e $7^{\circ}$ na curva lombar $\left(\right.$ de $23^{\circ}$ para $16^{\circ}$ ).
Após 2 anos atingido a maturidade esquelética e com 6 meses de tratamento, os ângulos de Cobb eram $21^{\circ}$ e $15^{\circ}$, respectivamente.

A correção da coluna através das técnicas de quiropraxia mostraram efeitos positivos, portanto, uma correção direita da coluna é essencial no tratamento da coluna.

Dos pacientes tratados através das técnicas quiropráticas, 90\% conseguiram uma correção ou estabilidade da curva.

Houve relato de alívio da dor, melhora na mobilidade, paciente mais confiante e visivelmente melhor em tônus muscular, além da eficácia na diminuição na curvatura da coluna vertebral. 
Tabela 1 (cont.). Apresentação das informações contidas nos artigos selecionados na Revisão Integrativa.

TÍTULO

Reduction of progressive thoracolumbar adolescent idiopathic scoliosis by chiropractic biophysics $^{\circledR} \quad\left(\mathrm{CBP}^{\circledR}\right)$ mirror image $\AA$ methods following failed traditional chiropractic treatment: case report ${ }^{4}$.

Changes in radiographic parameters following chiropractic treatment in 10 patients with adolescent idiopathic scoliosis: A retrospective chart review $^{18}$

INTERVENÇÃO

Realizaram escala para avaliar a dor através dos questionários IDH e ODI; Houve palpação da musculatura paravertebral direita seguido de avaliação postural; $\mathrm{Na}$ aplicação exclusiva da técnica $\mathrm{CBP}$, foram realizadas radiografias. No consultório, realizou-se os exercícios específico na PowerPlate, exercício de Fit Stik Pro Bar e recebeu tração em casa e no consultório, Aplicou-se terapia manipuladora espinhal em todas as área cervical, torácica e lombar bilateralmente;

As sessões foram praticadas de duas a três veze por semana e constituia em massagens, tração espinhal distração-flexão e manipulação da coluna vertebral. Continuou o tratamento até atingi a melhora máxima ou até a melhora nivelada do paciente.
RESULTADOS

Ao longo de 15 semanas, realizou-se 24 tratamentos em consultório, além de 45 sessões de bloqueio domiciliar ao decorrer desse período. Notou-se a redução da curva de escoliose toracolombar de $27^{\circ}$ para $8^{\circ}$, além do relato de melhora nos sintomas e aumento na pontuação quanto a melhora da dor.

Ocorreu durante um período médio de 10,3 meses. Observou-se melhora significativa das deformidades das curvas a partir do ângulo de Cobb pré-tratamento de média de $29,7^{\circ}$ até média de $23,4^{\circ}$, no processo final a correção geral foi de média de $21,2 \%$.

\section{CONCLUSÃO}

Houve relato de melhora de dor e de sintomas, além da redução de 19 graus na curvatura da coluna vertebral. Ainda assim, como mais de um tratamento foi realizado: tração, exercícios e bloqueio, é necessário maiores estudos/séries de caso para diferentes idades, tipos de curvas e magnitudes.

A partir do estudo de manipulação quiroprática em todos os pacientes, permitiu-se considerar as alterações radiológicas positivas. é a solução para impedir que o avanço da escoliose se desenvolva ou piore e assim seja fornecido o tratamento mais adequado ${ }^{15}$.

As técnicas quiropráticas consistem em manipulação espinhal, estimulação elétrica e exercícios ${ }^{7}$.A avaliação física básica estabelece uma avaliação visual do paciente conforme flexiona para a frente ${ }^{11}$. Na observação, percebe-se no paciente a presença da assimetria do tronco, que é proeminente na curva para a frente. Devido nessa posição a corcunda costal ou lombar torna-se mais evidente ${ }^{8}$. Na aplicação dessas manobras, uma área específica das vértebras e articulações é identificada, e um estímulo rápido e de baixa amplitude é aplicado para corrigir a articulação, aplicando força a ela para que a articulação se mova para a faixa fisiológica e além da faixa elástica, que é a faixa de movimento normal da articulação. Sendo assim, essas manipulações espinhais são conhecidas por corrigir uma coluna torcida, restaurar a desordem muscular, ajudar na restauração funcional dos nervos espinhais, e estimular o órgão tendinoso de Golgi localizado ao redor dos tendões para relaxar o músculo e expandir a amplitude de movimento ${ }^{6}$.

$\mathrm{O}$ resultado foi mais efetivo quando a quiropraxia é atrelada a exercícios específicos como tração repetitiva motorizada, distração da flexão, entre outros, que auxiliam no ganho de amplitude de movimento, bem como o uso concomitante de órteses ${ }^{16}$. Outrossim, as manobras quiropráticas auxiliam na melhora da qualidade de vida dos pacientes ${ }^{17}$ e com base na limitação dos movimentos espinhais na escoliose, o fortalecimento muscular ao redor da coluna e a manipulação dos tecidos moles se tornam essenciais para um melhor ajuste das vértebras ${ }^{6}$.

Conforme as recomendações do SOSORT (Sociedade sobre Escoliose Ortopédica e Tratamento de Reabilitação), a maior parte das crianças com escoliose leve $\left(<20^{\circ}\right)$ passará apenas por exames periódicos, em observação caso ocorra progressão. Entretanto, é essencial tratar a escoliose nos estágios iniciais da puberdade. Os indícios mais recentes comprovam que o risco de progressão no começo da puberdade é de $20 \%$ em $10^{\circ}$ de escoliose, $60 \%$ em $20^{\circ}$ de escoliose, e até $90 \%$ em $30^{\circ}$ de escoliose. Quando correlacionadas a uma coluna madura, a coluna pediátrica possui componentes mais cartilaginosos, centros de ossificação secundários e alusiva frouxidão dos ligamentos e articulações, a coluna imatura é, consequentemente, mais delicada ao tratamento conservador ${ }^{18}$.

A utilização do raio-X na prática clínica possui uma extensa relação com a quiropraxia. Os recentes conhecimentos de raios- $\mathrm{X}$ foram adicionados nos exames clínicos quiropráticos a partir de 1910, com o propósito declarado de visualizar o alinhamento das vértebras espinhais e um tratamento mais eficiente e direto ${ }^{19}$. O método de avaliação radiográfica é essencial para identificar se existem fraturas de compressão vertebral torácica que limitarão o potencial de correção ${ }^{20}$, além de servir para indicar a extensão da curva, diminuir o diagnóstico de outras possíveis causas (por exemplo, tumores), sendo normalmente efetuada durante a primeira visita ${ }^{11}$.Os diagnósticos de escoliose por meio de raios-X, são geralmente realizados da coluna completa com o paciente posicionado em pé ${ }^{8}$. Assim, por meio dos exames radiológicos, permitiu-se adquirir um maior resultado em adolescentes mais jovens e com 
curvas iniciais/moderadas, sendo as correções menos evidentes em adolescentes tardios com curvas mais desenvolvidas ${ }^{18}$.

Para garantir um êxito maior no tratamento, é exequível um tempo mínimo de 40 minutos por sessão, pelo menos por duas semanas, a julgar a condição de cada paciente. A quantidade de sessões também varia de 3 sessões por semana a 21 e o paciente precisa ser acompanhado até o fim da maturidade esquelética ${ }^{15}$.

As limitações deste estudo estão relacionadas à quantidade reduzida de publicações que utilizam essencialmente a quiropraxia no tratamento da Escoliose Idiopática do Adolescente (EIA). Vale ressaltar ainda, que os estudos apresentaram desenhos heterogêneos com pequenas amostras, diante disso, não se pode comprovar os reais efeitos desta intervenção neste público.

\section{Considerações Finais}

Com base no que foi exposto, a quiropraxia apresenta notáveis resultados a partir do primeiro mês de tratamento, no entanto, não foi possível concluir sobre a eficácia desta intervenção devido a quantidade reduzida e a heterogeneidade dos estudos. Com isso, espera-se que outras análises possam ser realizadas com o intuito de preencher esta lacuna científica e propagar os resultados sobre a quiropraxia.

\section{Referências Bibliográficas}

1. Pialasse JP, Simoneau M. Effectofbracingorsurgicaltreatmentson balance control in idiopathicscoliosis: three case studies. J CanChiropr Assoc. 2014;58(2):131-140.

2. Horne JP, Flannery R, Usman S. Adolescentidiopathicscoliosis: diagnosisand management. Am FamPhysician; 89(3):193-8. Feb.2014. PMID: 24506121.

3. Jada A, Mackel CE, Hwang SW, Samdani AF, Stephen JH, Bennett JT, et al. (2017). Evaluationand management ofadolescentidiopathicscoliosis: a review. Neurosurg Focus. 2017 [cited 2021 Mar 3];43(4). 2. ed. Availablefrom:

https://doi.org/10.3171/2017.7.FOCUS17297.

4. Haggard JS, Haggard JB, Oakley PA, Harrison DE Reduction of progressivet hora columbar adolescent idiopathics coliosis by chiropractic bio physics ${ }^{\circledR}(\mathrm{CBP} \circledast)$

5. Kotwicki T, Chowanska J, Kinel E, Czaprowski D, Tomaszewski M, Janusz P. Optimal management ofidiopathicscoliosis in adolescence. Adolesc Health Med Ther. 2013 Jul;4:59-73. doi: 10.2147/AHMT.S32088. PMID: 24600296 ; PMCID: PMC3912852.

6. Byun S, Han D. The effectofchiropractictechniquesonthe Cobb angle in idiopathicscoliosisarising in adolescence. J PhysTherSci. 2016; 28(4), p. 1106-1110.

7. Azham SZ, Kamaruddin NF, Afindi EE, Sallehudin LA, Haron MN, Rozali KN, et al. (2017). OutcomeofChiropracticTherapy in IdiopathicScoliosis - A PreliminaryStudy.J Clin Health Sci. 2017; 2(2), p. 26-30.

8. Weiss HR, Moramarco MM, Borysov M, Ng SY, Lee SG, Nan X, et al. Postural Rehabilitation for AdolescentIdiopathicScoliosisduringGrowth.
AsianSpine J. 2016 Jun;10(3):570-81. doi: 10.4184/asj.2016.10.3.570. Epub 2016 Jun 16. PMID: 27340540; PMCID: PMC4917779.

9. St-Georges M, Teles AR, Rabau O, Saran N, Ouellet JA, Ferland CE. Adolescentidiopathicscoliosis: evaluatingperioperativebackpainthrough a simultaneousmorphologicalandbiomechanical approach. BMC MusculoskeletDisord .21, 466 (2020). https://doi.org/10.1186/s12891-02003462-4.

10. Weinstein SL. MD. The Natural HistoryofAdolescentIdiopathicScoliosis. J PediatrOrthop. 2019; 39, p S44S46. doi: 10.1097/BPO.0000000000001350.

11. Lotan S, Kalichman L. Manual therapytreatment for adolescentidiopathicscoliosis. J BodywMovTher. 2019; 23 (1), pp. 189-193.

12. Morningstar M, Oslin D. Chiropracticrehabilitation plus nighttimebracing for progressiveadolescentidiopathicscoliosis: a casecontrolled series. ClinPract. 2019; 9 (4).

13. Woggon AJ, Woggon DA. Patient-reportedsideeffectsimmediatelyaft erchiropracticscoliosistreatment: a cross-sectionalsurveyutilizing a practicebasedresearch network. Scoliosis. 2015; 10 (29). Availablefrom: https://doi. org/10.1186/s13013-015-0053-8.

14. MorningstarMW, StitzelCJ, Siddiqui A, Dovorany B ChiropracticTreatments for IdiopathicScoliosis: A Narrative Review Basedon SOSORT OutcomeCriteria. J Chiropr Med. 2017a; 16(1), p. 64-71.

15. Chu EC, Huang KH.Bridgingthe gap betweenobservationandbracetreatment for adolescentidiopathicscoliosis. J Family Med PrimCare. 2017; 6 (2), p. 447.

16. Dovorany B, MorningstarMW, StitzelCJ, Siddiqui A. Resultsofchiropracticscoliosisrehabilitationtreatmentattwoyears postskeletalmaturity in identicalfemaletwins. J BodywMovTher. 2015; 19 (4), p. $592-596$

17. MorningstarMW, Dovorany B, StitzelCJ, Siddiqui. Chiropracticrehabilitation for adolescentidiopathicscoliosis: end-ofgrowthandskeletalmaturityresults. ClinPract. 2017 b; 7 (1), p.911.

18. Chu EC, Chakkaravarthy DM, Huang KH, Ho VW, Lo FS, Bhaumik A. Changes in radiographicparametersfollowingchiropractictreatment in 10 patientswithadolescentidiopathicscoliosis: A retrospectivechart review. ClinPract. 2020; 10(3) p.1258.

19. Jenkins HJ, Downie AS, Moore CS, French SD.Currentevidence for spinal X-ray use in thechiropracticprofession: a narrative review. Chiropr Man Therap. 2018; 26(48).

20. Oakley PA, Ehsani NN, Harrison DE. RepeatRadiography in MonitoringStructuralChanges in theTreatmentofSpinalDisorders in Chiropracticand Manual Medicine Practice: EvidenceandSafety. J Index Met. 2019; 17(4). 